\title{
THE SOLUTION OF INTEGRAL EQUATIONS
}

\author{
By A. C. Dixon.
}

[Received October 10th, 1908._-Read November 12th, 1908.-Revised February 9th, 1909.]

IN a paper by H. Schmidt (Wath. Ann., 64) a method of solving integral equations is given which is closely related to one given by me some years ago* for the case of analytical functions; this latter is in the present paper ( $\$ 2$ ) extended to more general functions. The results are not as a rule new, $t$ at any rate in form, but by means of a theorem given by Schmidt it is possible to use directly some of the late results in the theory of Fourier series, and so to remove the restriction of continuity which has often been put upon the functions involved. It appears, in fact, from the work of Lebesgue, referred to by Hobson (Functions of a Real Variable, p. 715, footnote), that the results apply at once to all limited summable functions, that is, pending the discovery of an unsummable function, to all known functions which do not become infinite.

1. Take the equation

$$
f(x)-\int_{0}^{1} \phi(x, y) f(y) d y=g(x),
$$

where $x$ is a real variable, ranging from 0 to $1, g$ and $\phi$ are known functions, and $f$ is the unknown function; the necessary restrictions on the nature of these functions are to be considered later ( $(3)$. The equation (1) may be regarded as a system of an infinite number of equations with an infinite number of unknowns, namely, the values of $f(x)$ for different values of $x$.

Let $\int_{1}^{1} \phi(x, y) f(y) d y$, which is a function of $x$ derived from $f(x)$ by a certain process, be denoted by $M f(x)$, so that $M$ is an operator transform-

* Camb. Plizl. Trans., Vol. xix., Part 2, pp. 208-210.

† For references see Mr. Bateman's paper, Proceedings, Ser. 2, Vol. 4, pp. 90-115. To his list may be added a further paper by Hilbert (Gött. Nachr., 1906), and others by Mercer (Camb. Phil. Trans., 1908), B. Heywood (Lionville's Journal, 1908), Schmidt (Math. Annalen, 63, 64, 65), My ller-Lebedeff (Math. Ann., 64). 
ing $f$ and analogous to a matrix in ordinary algebra. This operation can be repeated, so that we have

$$
M^{2} f(x)=\int_{0}^{1} \phi(x, y) M f(y) d y=\int_{0}^{1} \int_{0}^{1} \phi(x, y) \phi(y, t) f(t) d t d y,
$$

and 80 on.

The equation (1) is

$$
f(x)-M f(x)=g(x),
$$

from which it follows that

$$
M f(x)-M^{2} f(x)=M g(x),
$$

since $M$ is distributive, and similarly

$$
M^{2} f(x)-M^{3} f(x)=M^{2} g(x), \ldots
$$

Hence, by addition,

$$
f(x)=g(x)+M g(x)+M^{2} g(x)+\ldots+M^{n} g(x)+M^{n+1} f(x) .
$$

The last term here will tend to zero as $n$ increases indefinitely, if $f(x)$ is a limited function, and if we know that the greatest value of $M f(x)$ is, for all functions $f$, less numerically than $e$ times the greatest value of $f(x)$ where $e$ is $<1$. This is the case if

$$
\int_{0}^{1}|\phi(x, y)|^{2} d y<e^{2}
$$

for all values of $x$, since

$$
\left|\int_{0}^{1} \phi(x, y) f(y) d y\right|^{2} \leqslant \int_{0}^{1}|\phi(x, y)|^{2} d y \int_{0}^{1}|f(y)|^{2} d y .
$$

Hence, if $\quad \int_{0}^{1}|\phi(x, y)|^{2} d y<e^{2}$ for all values of $x$,

the only solution of (1) in which $f(x)$ is always finite is

$$
f(x)=g(x)+M g(x)+M^{2} g(x)+\ldots \text { to } \infty .
$$

This is Neumann's method.

For the condition (3) we may substitute the much less exacting one

$$
\int_{0}^{1} \int_{0}^{1}|\phi(x, y)|^{2} d x d y\left(=e^{2}\right)<1 \text {.* }^{*}
$$

\footnotetext{
* Schmidt, Math. Anı., 64, p. 163.
} 
For $\quad M^{n} f(x)=\int_{0}^{1} \int_{0}^{1} \ldots \phi\left(x, y_{1}\right) \phi\left(y_{1}, y_{2}\right) \ldots \phi\left(y_{n-1}, y_{n}\right) f\left(y_{n}\right) d y_{n} \ldots d y_{1}$

$$
=\int_{0}^{1} \int_{0}^{1} \phi\left(x, y_{1}\right) \phi_{n-1}\left(y_{1}, y_{n}\right) f\left(y_{n}\right) d y_{n} d y_{1}
$$

where $\phi_{n-1}\left(y_{1}, y_{n}\right)=\int_{0}^{1} \int_{0}^{1} \ldots \phi\left(y_{1}, y_{2}\right) \phi\left(y_{2}, y_{3}\right) \ldots \phi\left(y_{n-1}, y_{n}\right) d y_{n-1} \ldots d y_{2}$

$$
=\int_{0}^{1} \phi\left(y_{1}, u\right) \phi_{n-2}\left(u, y_{n}\right) d u \text {. }
$$

Thus

$$
\int_{0}^{1} \int_{0}^{1}\left|\phi_{n-1}\left(y_{1}, y_{n}\right)\right|^{2} d y_{n} d y_{1}
$$

$\leqslant \int_{0}^{1} \int_{v}^{1} \int_{0}^{1} \int_{0}^{1}\left|\phi\left(y_{1}, u\right) \phi_{n-2}\left(u, y_{n}\right) \phi\left(y_{1}, v\right) \phi_{n-2}\left(v, y_{n}\right)\right| d u d v d y_{n} d y_{1}$

$\leqslant \frac{2}{2} \int_{0}^{1} \int_{0}^{1} \int_{0}^{1} \int_{0}^{1}\left\{\left|\phi\left(y_{1}, u\right) \phi_{n-2}\left(v, y_{n}\right)\right|^{2}+\left|\phi\left(y_{1}, v\right) \phi_{n-2}\left(u, y_{n}\right)\right|^{2}\right\} d u d v d y_{n} d y_{1}$, which last expression $=e^{2} \int_{0}^{1} \int_{0}^{1}\left|\phi_{n-2}\left(v, y_{n}\right)\right|^{2} d v d y_{n}$.

Hence it follows that

$$
\int_{0}^{1} \int_{0}^{1}\left|\phi_{n-1}\left(y_{1}, y_{n}\right)\right|^{2} d y_{n} d y_{1} \leqslant e^{2 n-2}
$$

Now $\left|M I^{n} f(x)\right|^{2} \leqslant \int_{0}^{1} \int_{0}^{1}\left|\phi\left(x, y_{1}\right) f\left(y_{n}\right)\right|^{2} d y_{n} d y_{1} \int_{0}^{1} \int_{0}^{1}\left|\phi_{n-1}\left(y_{1}, y_{n}\right)\right|^{2} d y_{n} d y_{1}$

$$
\leqslant e^{2 n-2} \int_{0}^{1}|\phi(x, y)|^{2} d y \int_{0}^{1}|f(y)|^{2} d y .
$$

Thus $M^{\prime \prime} f(x)$ tends to zero when $n$ tends to infinity, and the method still holds good.

If $g(x)$ is a limited function, $e^{-n} M^{n} g(x)$ is finite for all values of $n$ and $x$, therefore the series (4) is absolutely and uniformly convergent: applying the operation $1-M$ to it, we see that it satisfies (1).

2. This solution may fail unless

$$
\int_{0}^{1} \int_{0}^{1}|\phi(x, y)|^{2} d x d y<1
$$

In a case of failure we proceed as follows.

We may put

$$
\phi=\phi_{11}+\phi_{12}+\phi_{21}+\phi_{22}
$$

where $\phi_{11}+\phi_{12}$ is the sum of the first $n$ terms of the Fourier sine-series derived from $\phi$ as a function of $x, \phi_{11}+\phi_{21}$ the sum of the first $n$ terms of 
the Fourier sine-series derived from $\phi$ as a function of $y, \phi_{11}$ is the sum of the terms in the first $n$ rows and columns of the double sine-series derived from $\phi$. That is,

$$
\begin{aligned}
& \phi_{11}(x, y)=4 \sum_{p=1}^{n} \sum_{\eta=1}^{n} \sin p \pi x \sin q \pi y \int_{0}^{1} \int_{0}^{1} \phi(t, u) \sin p \pi t \sin q \pi u d t d u \\
& \phi_{12}(x, y)+\phi_{11}(x, y)=2 \sum_{p=1}^{n} \sin p \pi x \int_{0}^{1} \phi(t, y) \sin p \pi t d t \\
& \phi_{21}(x, y)+\phi_{11}(x, y)=2 \sum_{q=1}^{n} \sin q \pi y \int_{0}^{1} \phi(x, u) \sin q \pi u d u
\end{aligned}
$$

the infinite series are not used, but $\phi_{22}$ is given as

$$
\phi-\left(\phi_{12}+\phi_{11}\right)-\left(\phi_{21}+\phi_{11}\right)+\phi_{11} \text {. }
$$

Let $g=g_{1}+g_{2}, f=f_{1}+f_{2}$ similarly, so that

$$
\begin{aligned}
& g_{1}(x)=2 \sum_{p=1}^{n} \sin p \pi x \int_{0}^{1} g(t) \sin p \pi t d t, \\
& f_{1}(x)=2 \sum_{p=1}^{n} \sin p \pi x \int_{0}^{1} f(t) \sin p \pi t d t=\sum_{1}^{n} \alpha_{p} \sin p \pi x, \text { say. }
\end{aligned}
$$

Then

$$
\int_{0}^{1} f_{2}(x) \sin p \pi x d x=0 \quad(p=1,2, \ldots, n) ;
$$

and therefore

$$
\int_{0}^{1} f_{2}(y) \phi_{11}(x, y) d y=0
$$

there being similar results for the products $f_{2} \phi_{21}, f_{1} \phi_{12}, f_{1} \phi_{22}$. Hence the equation (1) becomes

$$
\begin{aligned}
f_{1}+f_{2}-g_{1}-g_{2} & =\int_{0}^{1}\left(f_{1}+f_{2}\right)\left(\phi_{11}+\phi_{12}+\phi_{21}+\phi_{22}\right) d y \\
& =\int_{0}^{1}\left(f_{1} \phi_{11}+f_{1} \phi_{21}+f_{2} \phi_{12}+f_{2} \phi_{22}\right) d y .
\end{aligned}
$$

Multiply by $\sum_{p=1}^{n} \sin p \pi x \sin p \pi t$, and integrate with respect to $x$ from 0 to 1 , and put $x$ for $t$, then

$$
f_{1}(x)-g_{1}(x)=\int_{0}^{1}\left\{f_{1}(y) \phi_{11}(x, y)+f_{2}(y) \phi_{12}(x, y)\right\} d y
$$

and therefore, by subtraction,

$$
f_{2}(x)-g_{2}(x)=\int_{0}^{1}\left\{f_{1}(y) \phi_{21}(x, y)+f_{2}(y) \phi_{22}(x, y) ; d y .\right.
$$


Now (6) may be written

$$
\begin{aligned}
f_{2}(x)-\int_{0}^{1} \phi_{22}(x, y) f_{2}(y) d y & =g_{2}(x)+\int_{0}^{1} \phi_{21}(x, y) f_{1}(y) d y \\
& =g_{2}(x)+\sum_{p=1}^{n} a_{p} \int_{0}^{1} \phi_{21}(x, y) \sin p \pi y d y,
\end{aligned}
$$

and gives, by the method of $\S 1$, if

$$
\begin{gathered}
\int_{0}^{1} \int_{0}^{1}\left|\phi_{22}(x, y)\right|^{2} d x d y<1, \\
f_{2}(x)=\left(1+M_{22}+M_{22}^{2}+\ldots\right) \eta_{i} g_{2}(x)+\sum_{p=1}^{n} \alpha_{p} M M_{21} \sin p \pi x !,
\end{gathered}
$$

where $M_{22}, \ldots$ are related to $\phi_{92}, \ldots$ as $M$ to $\phi$. This may be written

$$
f_{2}(x)=\chi(x)+\sum_{i=1}^{n} a_{i}, \lambda_{p}(x)
$$

where

$$
\chi(x)=\left(1+i Y_{22}+M_{2 ! 2}^{2}+\ldots\right) g_{2}(x),
$$

and

$$
\lambda_{r}(x)=\left(1+M M_{22}+M I_{22}^{2}+\ldots\right) M_{21} \sin p \pi x .
$$

Substituting this value for $f_{2}(x)$ and $\Sigma a_{p} \sin p \pi x$ for $f_{1}(x)$ in (5), we have just $n$ linear equations to determine $a_{1}, a_{2}, \ldots, a_{n}$, since the coefficient of $\sin p \pi x$ must vanish for each value of $p$. If $\Delta$, the determinant of the coefficients of $a_{1}, a_{2}, \ldots, a_{n}$ in these equations, is not zero, then $a_{1}, a_{2}, \ldots$ are determined uniquely, and so is $f(x)$, which is equal to

$$
f_{1}(x)+f_{2}(x)
$$

that is, to

$$
\left.\chi(x)+\sum_{1}^{n} a_{p} ; \sin p \pi x+\lambda_{i^{\prime}}(x)\right\} .
$$

Since the process is reversible, the equations (5), (6) are both satisfied, and the method establishes the existence of a unique solution of (1), namely $f(x)$, subject to the condition $\Delta \neq 0$, and to restrictions on the nature of the functions, which. will now be discussed.

3. For the success of the operations in $\S 1$ it is necessary that $M g(x), M^{2} g(x), \ldots$ have meanings, so that $\phi(x, y) g(y)$ must be summable in $y, \phi(x, y) \phi(y, t) g(t)$ in $y, t$, and so on.

As none but summable functions are known, there appears to be no use here in going behind the assumption that all functions are summable. IVe shall also suppose the functions $g, \phi$ limited, and thus the order of integration will be indifferent in repeated integrals. (Hobson, Functions of a Real Variable, p. 581.) Also, in $\$ 1$, the value found for $f$ will be limited. 
Since the functions are limited and summable, the Fourier coefficients in $\S 2$ exist. We need to be able to choose $n$ so that

$$
\int_{0}^{1} \int_{0}^{1}\left|\phi_{22}(x, y)\right|^{2} d x d y<1
$$

Now, as $n$ increases without limit,

$$
\int_{0}^{1} \int_{0}^{1}\left|\phi_{22}(x, y)\right|^{2} d x d y
$$

decreases, tending towards a limit, which is, according to Lebesgue's results, 0 in all known cases. ${ }^{*}$

For the success of the present method it is only necessary that this limit be $<1$; this supposition as to the nature of $\phi$ will be made.

The only further condition to be satisfied is that the determinant $\Delta$ do not vanish, and this ensures the existence and uniqueness of the solution. In particular, when $g(x)=0$, we have $f(x)=0$.

4. It is important to discuss what happens when $\Delta=0$. The system of equations given by (5) is

$$
\begin{aligned}
& \alpha_{p}-2 \int_{0}^{1} g_{1}(x) \sin p x \pi d x \\
& =2 \Sigma \alpha_{q} \int_{0}^{1} \int_{0}^{1} \sin p x \pi \sin q y \pi \phi_{11}(x, y) d y d x \\
& +2 \int_{0}^{1} \int_{0}^{1} \chi(y) \phi_{12}(x, y) \sin p x \pi d y d x \\
& +2 \Sigma \alpha_{q} \int_{0}^{1} \int_{0}^{1} \lambda_{q}(y) \phi_{12}(x, y) \sin p x \pi d y d x \quad(p=1,2, \ldots, n),
\end{aligned}
$$

* Hobson (Functions of a Real Variable, p. 715, note) gives the result for a simple Fourier series; hence, in the present case, if $\phi$ is real,

$$
\int_{0}^{1}\left\{\phi_{21}(x, y)+\phi_{22}(x, y)\right\}^{2} d x
$$

tends to zero as $n$ increases, for all values of $y$. If $n$ is chosen so great that this integral is $<1$ for all values of $y$, we have

$$
\begin{array}{ll} 
& \int_{0}^{1} \int_{0}^{1}\left(\phi_{21}+\phi_{22}\right)^{2} d x d y<1, \\
\text { and still more } & \int_{0}^{1} \int_{0}^{1}\left(\phi_{22}\right)^{2} d x d y<1, \\
\text { since } & \int_{0}^{1} \phi_{21} \phi_{22} d y=0:
\end{array}
$$

the extension to a complex $\phi$ is immediate. 
or $\alpha_{1}-2 \Sigma \alpha_{\alpha_{1}} \int_{0}^{1} \int_{0}^{1} \sin p x \pi\left\{\sin q y \pi \phi_{11}(x, y)+\lambda_{q}(y) \phi_{12}(x, y)\right\} d y d x$

$$
\begin{array}{r}
=2 \int_{0}^{1} g_{1}(x) \sin p x \pi d x+2 \int_{0}^{1} \int_{0}^{1} \chi(y) \phi_{12}(x, y) \sin p x \pi d y d x \\
(p=1,2, \ldots, n) .
\end{array}
$$

Here

$$
\chi(x)=g_{2}(x)+M_{22} g_{2}(x)+M_{22}^{2} g_{2}(x)+\ldots,
$$

where $M_{22}$ differs from $M$ only in that $\phi_{22}$ takes the place of $\phi:$ in what follows $M_{12}, N_{11}, M_{21}$ are used similarly.

If $g(x)=0$, the second members in (7) are all zero, but since $\Delta=0$, the equations have a solution, say

$$
\beta_{1}, \beta_{2}, \ldots, \beta_{n} \text {. }
$$

Then, if

$$
\begin{gathered}
h(x)=\sum_{p=1}^{n} \beta_{p}\left\{\sin p x \pi+\lambda_{p}(x)\right\}, \\
h(x)=M h(x),
\end{gathered}
$$

we have

so that when $g(x)=0, f(x)$ is not necessarily zero, but may be any multiple of $h(x)$.

To verify (8), put $h_{1}(x), h_{2}(x)$ for $\Sigma \beta_{p}$ sin $p x \pi$ and $\Sigma \beta_{p} \lambda_{p}(x)$ respectively. In (7), multiply by $\sin p x \pi$ and sum for $p=1,2, \ldots, n$, taking $g(x)=0$; thus, since $\alpha_{1}$, is replaced by $\beta_{p}$,

$$
h_{1}(x)-\int_{0}^{1} h_{1}(y) \phi_{11}(x, y) d y-\int_{0}^{1} h_{2}(y) \phi_{12}(x, y) d y=0,
$$

or

$$
h_{1}(x)=M_{11} h_{1}(x)+M_{12} h_{2}(x) .
$$

Again, the equation defining $\lambda_{p}(x)$ is $(\$ 2)$

$$
\left(1-M_{22}\right) \lambda_{p}(x)=\int_{0}^{1} \phi_{21}(x, y) \sin p y \pi d y
$$

and therefore $\quad\left(1-M_{22}\right) h_{2}(x)=\int_{0}^{1} \phi_{21}(x, y) h_{1}(y) d y$,

or

$$
h_{2}(x)=M_{21} h_{1}(x)+M_{22} h_{2}(x) .
$$

Adding (9), (10), we have (8), since $M_{11} h_{2}(x), M_{12} h_{1}(x), M_{21} h_{2}(x)$, $M_{22} h_{1}(x)$ are zero.

If all the $(p-1)$-th minors of $\Delta$ vanish, but not all its $p$-th minors, there are exactly $p$ linearly independent sets, such as

$$
\beta_{1}, \beta_{2}, \ldots, \beta_{n} \text {, }
$$

which, when substituted for $\alpha_{1}, \alpha_{2}, \ldots, \alpha_{n}$,

make the first members vanish in the equations (7), and therefore there 
are $p$ linearly independent functions such as $h(x)$, satisfying (8). This case is more fully discussed below $(\S 8 a)$.

5. Again, if $\Delta=0$, but its first minors are not all zero, there are $n$ constants $\kappa_{1}, \kappa_{2}, \ldots, \kappa_{n}$, such that if the left sides in (7) are multiplied by them respectively, the sum of the products is zero, and the equations are soluble if the same is true for the right sides, that is, if

$$
\int_{0}^{1} g_{1}(x) k_{1}(x) d x+\int_{0}^{1} \int_{0}^{1} \chi(y) \phi_{12}(x, y) k_{1}(x) d y d x=0,
$$

where

$$
k_{1}(x)=\sum_{p=1}^{n} \kappa_{p} \sin p x \pi \text {. }
$$

The second term in (11), when transformed by writing for $\chi$ its value $g_{2}+M_{22} g_{2}+\ldots$, and by reversing the order of integration in each term, may be written

$$
\int_{0}^{1} g_{2}(x) k_{2}(x) d x
$$

where $\quad k_{2}(x)=\bar{M}_{12} k_{1}(x)+\bar{M}_{22} \bar{M}_{12} k_{1}(x)+\bar{M}_{22}^{2} \bar{M}_{12} k_{1}(x)+\ldots,{ }^{*}$

the bar indicating what corresponds to the interchange of rows and columns in a finite matrix, so that

$$
\bar{M} f(x)=\int_{0}^{1} \phi(y, x) f(y) d y .
$$

Since the integrals of $g_{1} k_{2}$ and $g_{2} k_{1}$ are zero, we may write (11) as

$$
\int_{0}^{1} g(x) k(x) d x=0,
$$

where

$$
k(x)=k_{1}(x)+k_{2}(x) \text {. }
$$

If, then, $\Delta=0$, but its first minors do not all vanish, the equation (1) can be solved when the function $g(x)$ satisfies the condition

$$
\int_{0}^{1} g(x) k(x) d x=0
$$

where $k(x)$ is a certain function of $x$.

If the $(p-1)$-th minors of $\Delta$ all vanish, the only difference is that $g(x)$ must satisfy $p$ conditions of this nature.

6. From (12), we have

$$
k_{2}(x)=\bar{M}_{12} k_{1}(x)+\bar{M}_{22} k_{2}(x),
$$

- The proof of convergency in $\S 1$ applies to this series also.

SER. 2. voL. 7, No. 1025. 
and another relation is found from the original property of $\kappa_{1}, \kappa_{2}, \ldots$, that when the left sides in (7) are multiplied by these quantities, the sum of the products vanishes independently of $\alpha_{1}, a_{2}, \ldots$, by putting

This result is

$$
\alpha_{p}=\sin p x \pi \quad(p=1,2, \ldots, n) .
$$

$$
k_{1}(x)=\int_{0}^{1} k_{1}(t) \phi_{11}(t, x) d t+2 \sum_{l=1}^{n} \sin q x \pi \int_{0}^{1} \int_{0}^{1} k_{1}(t) \lambda_{l l}(y) \dot{\phi}_{12}(t, y) d y d t .
$$

The second term on the right in (15) may be written

$$
2 \Sigma \sin q x \pi \int_{0}^{1} k_{1}(t) M M_{12} \lambda_{I_{1}}(t) d t
$$

or

$$
\begin{aligned}
& 2 \sum_{i=1}^{n} \sum_{r=0}^{\infty} \sin q x \pi \int_{0}^{1} k_{1}(t)\left(M_{12} M_{22}^{r} M_{21} \sin q t \pi\right) d t, \\
& 2 \sum_{i=1}^{n} \sum_{r=0}^{\infty} \sin q x \pi \int_{0}^{1} \sin q t \pi . \bar{M}_{21} \bar{M}_{22}^{r} \bar{M}_{12} k_{1}(t) d t,
\end{aligned}
$$

by reversing the order of the integrations involved, or

$$
\sum_{r=0}^{\infty} \bar{I}_{21} \bar{Y}_{2: 2}^{r} \bar{I}_{12} k_{1}(x)
$$

since $\bar{M}_{21}$ applied to any function gives a result of the form

$$
\sum_{p=1}^{n} a_{p} \sin p x \pi
$$

a rinite Fourier series. The second term on the right in (15), is thus no other than

and (15) becomes $\quad k_{1}(x)=\bar{M}_{11} k_{1}(x)+\bar{M}_{21} k_{2}(x)$.

Adding this to (14), we have $k(x)=\vec{M} k(x)$.

Hence when $د=0$, and its first minors do not all vanish, the equations

$$
f^{\prime}(x)=M f(x) \text { and } f(x)=\bar{M} f(x)
$$

can both be satisfied, namely, by the functions $h(x), k(x)$ respectively; the equation

$$
f(x)-M f(x)=g(x)
$$

can be satisfied, if

$$
\int_{0}^{1} g(x) k(x) d x=0
$$


and the solution is not unique on account of an arbitrary added. multiple of $h(x)$ : similarly* the equation

$$
f(x)-\bar{M} f(x)=g(x)
$$

can be satisfied, if $\quad \int_{0}^{1} g(x) h(x) d x=0$,

and the solution is not unique on account of an arbitrary added multiple of $k(x)$. If all the $(p-1)$-th minors of $\Delta$, but not all the $p$-th, vanish, there are $p$ functions in the place of each of the single functions $h, k$. The assumptions made do not exclude any known limited functions from the scope of the theory. The same may be said of Schmidt's solution.

7. If, treating $y$ as a parameter, we find by the methods of $\$ \$ 1,2$, a function $\psi$ satisfying the equation

we have, from (1),

$$
(1-\bar{M}) \psi(y, x)=\phi(y, x) \quad(\Delta \neq 0),
$$

$$
\begin{aligned}
\int_{0}^{1} \psi(y, x) g(x) d x & =\int_{0}^{1} \psi(y, x)(1-M) f(x) d x \\
& =\int_{0}^{1} f(x)(1-\bar{M}) \psi(y, x) d x \\
& =\int_{0}^{1} f(x) \phi(y, x) d x .
\end{aligned}
$$

Hence

$$
\int_{0}^{1} \psi(x, y) g(y) d y=M f(x)=f(x)-g(x),
$$

and the solution of (1) may be put in the form

$$
f(x)=g(x)+\int_{0}^{1} \psi(x, y) g(y) d y
$$

where $\psi$, the " solving function," does not depend on $g$.

If we write $x$ or $y$ as a suffix when the other is to be parametric, we have

and

$$
\begin{aligned}
M_{x} \phi(x, y) & =\bar{M}_{y} \phi(x, y), \\
N \Lambda_{x} \bar{M}_{y} \chi(x, y) & =\bar{M}_{y} M I_{x} \chi(x, y),
\end{aligned}
$$

* Of the four events : the vanishing of $\Delta$ and the corresponding determinant $\bar{\Delta}$, and the existence of $h(x), k(x)$, the occurrence of any one enables us to conclude that of the other three. All these results are well known. 
if $\chi(x, y)$ is limited and summable, so as to allow change of order in the double integral. Then, since

$$
\begin{aligned}
\left(1-\bar{M}_{y}\right) \psi(x, y) & =\phi(x, y), \\
\left(1-\bar{M}_{y}\right)\left(1-M_{x}\right) \psi(x, y) & =\left(1-M_{x}\right) \phi(x, y) \\
& =\left(1-\bar{M}_{y}\right) \phi(x, y), \\
\left(1-M_{x}\right) \psi(x, y) & =\phi(x, y),
\end{aligned}
$$

and

since a limited solution of such an equation as (1) is unique. Hence $\psi$ is also the solving function for the equation

$$
(1-\bar{M}) f(x)=g(x),
$$

whose solution may be written

$$
f(x)=g(x)+\int_{0}^{1} \psi(y, x) g(y) d y .^{*}
$$

8. The solution of the equation

$$
(1-M)^{2} f(x)=g(x)
$$

mas be carried out either by two applications of the method that has been given, or by a single one with the function $\phi(x, y)$ replaced by

$$
2 \phi(x, y)-\int_{0}^{1} \phi(x, t) \phi(t, y) d t .
$$

When $\lrcorner=0$, a distinction must be made between two cases as follows.

If

$$
\int_{0}^{1} g(x) k(x) d x=0
$$

the equation (1) has a solution, say

$$
f(x)=\lambda(x)+a h(x),
$$

where $a$ is an arbitrary constant. The equation

$$
(1-M) f(x)=\lambda(x)+a h(x)
$$

has a solution, if $\left.\quad \int_{0}^{1} i \lambda(x)+a h(x)\right\} k(x) d x=0$, and $a$ can be chosen uniquely so as to satisfy this, if

$$
\int_{0}^{1} h(x) h(x) d x \neq 0
$$


In this case, then, the same condition which ensures the solubility of

$$
(1-M) f(x)=g(x),
$$

ensures that of

$$
(1-M)^{2} f(x)=g(x) \text {, }
$$

and, in fact, of

$$
(1-M)^{p} f(x)=g(x),
$$

where $p=3,4,5, \ldots$ : the solution of (20) contains one arbitrary constant.

$$
\text { On the other hand, if } \int_{0}^{1} h(x) k(x) d x=0 \text {, }
$$

the determination of $a$ fails, and a second condition

$$
\int_{0}^{1} \lambda(x) k(x) d x=0
$$

is necessary for the solubility of (18).

On account of (21) we are now able to put

so that (22) becomes

$$
k(x)=(1-\bar{M}) k^{\prime}(x),
$$

$$
\begin{aligned}
0 & =\int_{0}^{1} \lambda(x)(1-\bar{M}) k^{\prime}(x) d x \\
& =\int_{0}^{1} k^{\prime}(x)(1-M) \lambda(x) d x=\int_{0}^{1} k^{\prime}(x) g(x) d x .
\end{aligned}
$$

The conditions (19) and (23) ensure the solubility of (18), and the solution now contains two arbitrary constants, and has, in fact, a complementary function

$$
a h^{\prime}(x)+a^{\prime} h(x),
$$

where $h^{\prime}(x)$ is given by $\quad(1-M) h^{\prime}(x)=h(x)$,

which is soluble on account of (21).

The same conditions, (13) and (23), ensure the solubility of (20), unless

$$
\int_{0}^{1} h(x) k^{\prime}(x) d x=0
$$

a condition which may be written

$$
\int_{0}^{1} h^{\prime}(x) k(x) d x=0,
$$

since these two quantities may be put in the forms

$$
\int_{0}^{1} k^{\prime}(x)(1-M) h^{\prime}(x) d x, \quad \int_{0}^{1} h^{\prime}(x)(1-\bar{M}) k^{\prime}(x) d x=0 .
$$


Hence the following theorem:-The number of conditions necessary and sufficient for the solubility of

$$
(1-M)^{p} f(x)=g(x) \quad \text { is } \quad p^{\prime} \leqslant p,
$$

and these conditions are

$$
\int_{0}^{1} l^{(r)}(x) g(x) d x=0 \quad\left(r=0,1, \ldots, p^{\prime}-1\right),
$$

where

$$
\begin{aligned}
(1-\bar{M}) k^{(r)}(x) & =k^{(r-1)}(x) \quad\left(r=1,2, \ldots, p^{\prime}-1\right) \\
& =0 \quad(r=0) ;
\end{aligned}
$$

if $p^{\prime}<p$, it is fixed as the lowest number for which

$$
\int_{0}^{1} h(x) k^{\left(p^{\prime}-1\right)}(x) d x \neq 0,
$$

so that the sequence of functions $k^{(0)}(x), k^{(1)}(x), \ldots$ cannot be continued beyond $k^{\left(p^{\prime}-1\right)}(x)$.

The number of conclitions for the solubility of

$$
(1-\bar{M})^{p} f(x)=g(x) \text { is also } p^{\prime},
$$

and these conditions are $\quad \int_{1}^{1} h^{(i)}(x) g(x) d x=0 \quad\left(r=0,1, \ldots, p^{\prime}-1\right)$,

where

$$
\begin{aligned}
(1-M) h^{(r)}(x) & =l^{(r-1)}(x) \quad\left(r=1,2, \ldots, p^{\prime}-1\right) \\
& =0 \quad(r=0) .
\end{aligned}
$$

The value of $\int_{11}^{1} h^{(r)}(x) k^{(s)}(x) d x$ is the same so long as $r+s$ is kept the same.

8a. If all the $(p-1)$-th minors of $\Delta$ vanish, but not all its $p$-th minors, there are, as pointed out in $\S \S 4-6$, two sets of $p$ functions each,

$$
\begin{array}{lll}
h_{1}, & h_{2}, \ldots, & h_{p}, \\
k_{1}, & k_{2}, \ldots, & k_{p} ;
\end{array}
$$

each set may be linearly transformed, so that we may suppose

$$
\int_{0}^{1} h_{\mathfrak{i}}(x) k_{j}(x) d x=0 \quad(i \neq j) .
$$

Then the solution of the equation (1) is possible, if

$$
\int_{0}^{1} g(x) k_{i}(x) d x=0 \quad(i=1,2, \ldots, p)
$$


and is of the form $\quad f(x)=\lambda(x)+\sum_{1}^{\prime \prime} a_{i} h_{i}(x)$.

No further conditions are necessary for the solubility of (18), if

$$
\int_{0}^{1} h_{i}(x) h_{i}(x) d x \neq 0 \quad(i=1,2, \ldots, p),
$$

but for each vanishing integral of this system one condition must be added ; suppose the number of vanishing integrals to be $q$, and the corresponding suffixes $1,2, \ldots, q$.

Then there will be two sets of $q$ functions each,

$$
\begin{aligned}
& k_{1}^{\prime}, k_{2}^{\prime}, \ldots, k_{\eta}^{\prime}, \\
& k_{1}^{\prime}, k_{2}^{\prime}, \ldots, k_{\eta}^{\prime},
\end{aligned}
$$

such that

$$
\left.\begin{array}{l}
(1-M) h_{i}^{\prime}=h_{i} \\
(1-\bar{M}) k_{i}^{\prime}=k_{i}
\end{array}\right\}, \quad(i=1,2, \ldots, q),
$$

and the new conditions are

$$
\int_{0}^{1} g(x) k_{i}^{\prime}(x) d x=0 \quad(i=1,2, \ldots, q) .
$$

The complementary function in the solution of (18) is

$$
\sum_{1}^{\prime \prime} a_{i} h_{i}^{\prime}+\sum_{1}^{\prime \prime} a_{i}^{\prime} h_{i} .
$$

Also

$$
\int_{0}^{1} h_{i}^{\prime}(x) h_{j}(x) d x \text { and } \int_{0}^{1} h_{i}(x) h_{i}^{\prime}(x) d x
$$

may be supposed to vanish when $j>q$, by fixing the arbitrary multiples of $h_{j}, k_{j}$ contrained in $h_{i}^{\prime}, k_{i}^{\prime}$ respectively.

The properties so far stated are not affected when $h_{1}, h_{2}, \ldots, h_{l}$ and $h_{1}^{\prime}, h_{2}^{\prime}, \ldots, h_{q}^{\prime}$, or $k_{1}, k_{2}, \ldots, k_{q_{1}}$ and $k_{1}^{\prime}, k_{2}^{\prime}, \ldots, k_{i,}^{\prime}$, are subjected to any the same linear transformation, which may be so chosen that

$$
\int_{0}^{1} h_{i}^{\prime}(x) k_{j}(x) d x, \text { which }=\int_{0}^{1} h_{i}(x) k_{j}^{\prime}(x) d x,
$$

is 0 , when $i \neq j$, or when $i=j=1,2, \ldots, r(r \leqslant q)$, and that these integrals do not vanish when $i=j=r+1, r+2, \ldots, q$.

Then $r$ is the number of further conditions necessary for the solubility of

$$
(1-M)^{8} f=g,
$$


and these conditions are

$$
\int_{0}^{1} g(x) k_{i}^{\prime \prime}(x) d x=0 \quad(i=1,2, \ldots, v),
$$

where

$$
(1-\bar{M}) k_{i}^{\prime \prime}=k_{i}^{\prime} \text {. }
$$

Since

$$
\int_{0}^{1} h_{i} k_{j} d x=0 \quad(j=1,2, \ldots, q),
$$

we may alter any, or all, of the functions $k_{1}^{\prime}$ by adding $\sum_{1}^{q} b_{j} k_{j}$, where $b_{j}$ is arbitrary : hence we may alter $k_{i}^{\prime \prime}$ by adding $\sum_{1}^{q} b_{j} k_{j}^{\prime}+\sum_{1}^{p} c_{j} k_{j}$. Here, when $j=q+1, q+2, \ldots, p$, we may choose $c_{j}$, so that

$$
\int_{0}^{1} h_{\mathrm{j}} k_{i}^{\prime \prime} d x=0
$$

and when $j=r+1, r+2, \ldots, q$, we may choose $b_{j}$, so that

$$
\int_{0}^{1} h_{j} k_{i}^{\prime \prime} d x=0
$$

and $c_{j}$, so that

$$
\int_{0}^{1} h_{j}^{\prime} k_{i}^{\prime \prime} d x=0 \text {. }
$$

The values of $b_{j}$ and $c_{j}$, when $j \leqslant r$ are still arbitrary.

Similarly we may suppose

$$
\begin{gathered}
\int_{0}^{1} k_{j} h_{i}^{\prime \prime} d x=0=\int_{0}^{1} k_{j}^{\prime} h_{i}^{\prime \prime} d x \quad(q \geqslant j>r), \\
\int_{0}^{1} k_{j} h_{i}^{\prime \prime} d x=0 \quad(j>q) .
\end{gathered}
$$

Then, by a simultaneous linear transformation of

$$
\left(k_{1}, \ldots, k_{r}\right)\left(k_{1}^{\prime}, \ldots, k_{r}^{\prime}\right)\left(k_{1}^{\prime \prime}, \ldots, k_{r}^{\prime \prime}\right) \text {, }
$$

we may make $\quad \int_{0}^{1} h_{i}^{\prime \prime} k_{j} d x=0 \quad(i \neq j$ or $i=j=1,2, \ldots, s)(s \leqslant r)$,

and

$$
\int_{0}^{1} h_{i}^{\prime \prime} k_{i} d x \neq 0 \quad(i=s+1, s+2, \ldots, r) .
$$

It is to be remembered that

$$
\int_{0}^{1} h_{i}^{\prime \prime} k_{j} d x=\int_{0}^{1} h_{i}^{\prime} k_{j}^{\prime} d x=\int_{0}^{1} h_{i} k_{j}^{\prime \prime} d x .
$$

There are now functions

$$
h_{i}^{m}, k_{i}^{m} \quad(i=1,2, \ldots, s) \text {, }
$$


and $k_{i}^{m}$ contains an arbitrary expression

$$
\sum_{1}^{r} b_{j} k_{j}^{\prime \prime}+\sum_{1}^{r} c_{j} k_{j}^{\prime}+\sum_{1}^{n} e_{j} k_{j},
$$

in which $e_{j}$ may be so chosen that

$$
\begin{aligned}
& \int_{0}^{1} k_{i}^{\prime \prime \prime} h_{j} d x=0 \quad(j>q), \\
& \int_{0}^{1} k_{i}^{\prime \prime} h_{j}^{\prime} d x=0 \quad(q \geqslant j>v), \\
& \int_{0}^{1} k_{i}^{\prime \prime} h_{j}^{\prime \prime} d x=0 \quad(r \geqslant j>s),
\end{aligned}
$$

and $b_{j}, c_{j}$, when $j>s$, may be so chosen that

$$
\int_{0}^{1} k_{i}^{\prime \prime \prime} h_{j} d x=\int_{0}^{1} k_{i}^{\prime \prime \prime} h_{j}^{\prime} d x=0 .
$$

The same treatment may be applied to $h_{i}^{\prime \prime \prime}$. And so on.

It will be found ( $(11)$ that this process must stop after a finite number of stages, and when it does so we have two sets of functions in canonical form, each set containing $p+q+r+\ldots$, and every function in each set having a mate in the other set, such that

$$
\int_{0}^{1} h k d x
$$

vaniskes when the functions $h, k$ are not mates, and does not vanish when they are. Each set can be divided into sequences, and the order of the functions in any sequence $\left(h, h^{\prime}, \ldots\right)$ is the reverse of the order of their mates in the corresponding sequence $\left(k, k^{\prime}, \ldots\right)$.

9. The above theory is unchanged, if we put $\theta M$ in the place of $M$, where $\theta$ is a parametric constant. The metbod of $\$ 1$ gives as the solution of

$$
\begin{gathered}
(1-\theta M) f(x)=g(x), \\
f(x)=g(x)+\theta M g(x)+\theta^{2} M^{2} g(x)+\ldots,
\end{gathered}
$$

that is, a power series in $\theta$ whose circle of convergency has a radius not less than the square root of the reciprocal of

$$
\int_{0}^{1} \int_{0}^{1}|\phi(x, y)|^{2} d x d y
$$


hence $f(x)$ is an analytical function of the complex variable $\theta$, at least within this circle.

If $\theta$ lies outside this circle, the method of $\$ 2$ has to be used, and $\chi(x)$, $\lambda_{p}(x)$ are analytical functions of $\theta$ within a wider circle, whose radius increases with $n$ : there is no superior limit to this radius if

$$
\lim _{n=\infty} \int_{0}^{1} \int_{0}^{1}\left|\phi_{32}\right|^{2} d x d y=0
$$

which assumption does not exclude any known functions. Thus $\Delta$ and also $\Delta f$ are functions of $\theta$ without singularity within this wider circle, and the singularities of $f$, for finite values of $\theta$, are all poles, possibly of different orders, at the zeros of $\Delta$ : except in the domain of such a zero $f$ is an analytical function of $\theta$.

10. Let $\theta_{1}$ be a zelo of $\Delta$ and $h(x), k(x)$ the associated functions, as before. Let the expansion of $\Delta f(x)$ in the domain of $\theta_{1}$ be

$$
\lambda(x)+\left(\theta-\theta_{1}\right) \lambda_{1}(x)+\left(\theta-\theta_{1}\right)^{2} \lambda_{2}(x)+\ldots
$$

Then $-\lambda(x)$ is equal to the determinant got when $\theta=\theta_{1}$ by bord ering $\Delta$ with an $(n+1)$-th column consisting of the right sides of $(7)$, and an $(n+1)$-th row in which the $p$-th member is $\sin p x \pi+\lambda_{p}(x)$; hence $\lambda(x)$ cannot vanish independently of $g(x)$ unless all the first minors of $\Delta$ are zero: for instance, when $g(x)=\sin p x \pi$, the value of $2 \int_{0}^{1} \lambda(x) \sin q x \pi d x$ is the minor got by leaving out the $p$-th row and $q$-th column: suppose then that these minors do not all vanish.

From the expansion

$$
\Delta f(x)=\lambda(x)+\left(\theta-\theta_{1}\right) \lambda_{1}(x)+\ldots
$$

we get, by the operation $1-\theta M$,

$$
\Delta g(x)=(1-\theta M) \lambda(x)+\left(\theta-\theta_{1}\right)(1-\theta M) \lambda_{1}(x)+\ldots .
$$

Here put $\theta=\theta_{1}$, so that $\Delta=0$; then

$$
\left(1-\theta_{1} M\right) \lambda(x)=0,
$$

that is, $\lambda(x)=a h(x)$, where $a$ is some constant and is not zero. Also

$$
(1-\theta M) \lambda(x)=\left(1-\frac{\theta}{\theta_{1}}\right) \lambda(x) .
$$


In (24), multiply by $k(x)$ and integrate. Thus

$$
\begin{aligned}
& \Delta \int_{0}^{1} g(x) k(x) d x \\
& \quad=\left(1-\frac{\theta}{\theta_{1}}\right) a \int_{0}^{1} h(x) k(x) d x+\left(\theta-\theta_{1}\right) \int_{0}^{1} k(x)(1-\theta M) \lambda_{1}(x) d x+\ldots
\end{aligned}
$$

The integral last written

$$
=\int_{0}^{1} \lambda_{1}(x)(1-\theta \bar{M}) k(x) d x=\left(1-\frac{\theta}{\theta_{1}}\right) \int_{0}^{1} \lambda_{1}(x) k(x) d x .
$$

So for the later terms : all terms on the right in (25) contain the factor $\left(\theta-\theta_{1}\right)^{2}$, except the first term. Thus, unless

$$
\int_{0}^{1} h(x) k(x) d x=0
$$

the zero of $\Delta$ is a simple one, and $\Delta^{-1} \lambda(x)$, the first term in the expansion of $f(x)$ in ascending powers of $\theta-\theta_{1}$, is

$$
\frac{h(x)}{1-\frac{\theta}{\theta_{1}}} \int_{0}^{1} h(x) k(x) d x .
$$

that is, $f(x)$ has a simple pole with the residue indicated.

11. When the first minors of $\Delta$ all vanish or:

$$
\int_{0}^{1} h(x) k(\dot{x}) d x=0
$$

the case is more complicated. Suppose that, when $\theta=\theta_{1}$, all the $(p-1)$-th minors of $\Delta$ vanish, but not all the $p$-th; then we can choose $n-p$ of the equations (7) whose left sides will be linearly independent when $\theta=\theta_{1}$. Using these, we can reduce the other $p$ equations so that the left sides will vanish when $\theta=\theta_{1}$, and will therefore have $\theta-\theta_{1}$ as a factor : this factor will not enter on the right unless $g(x)$ is restricted. When this factor is taken out from the left sides, the results may or may not be linearly independent of each other and of the former $n-p$ expressions when $\theta=\theta_{1}$; if they are not, then some of them may be reduced so as to contain the factor $\theta-\theta_{1}$ again, and so on. In this way the equations (7) can be replaced by a set in which $n-p$ of the left sides do not vanish when $\theta=\theta_{1}$,

$p_{1}$ of the left sides contain the factor $\theta-\theta_{1}$,

$\begin{array}{lllll}p_{2} & " & n & " & \left(\theta-\theta_{1}\right)^{2}, \\ p_{8} & " & n & n & \left(\theta-\theta_{1}\right)^{3},\end{array}$


and so on, and $p_{1}+p_{2}+p_{3}+\ldots=p$. After these factors have been taken away, the expressions are linearly independent when $\theta=\theta_{1}$ : also, no linear combination of the right sides, with constant coefficients, vanishes independently of $g(x)$.

Hence $\Delta$ contains $\theta-\theta_{1}$ to the power $p_{1}+2 p_{3}+3 p_{3}+\ldots$ and the expression for $f(x)$ will contain fractional terms with powers of $\theta-\theta_{1}$ in the denominator, and may be written

$$
\frac{\mu_{1}(x)}{\theta-\theta_{1}}+\frac{\theta \mu_{2}(x)}{\left(\theta-\theta_{1}\right)^{2}}+\ldots+\frac{\theta^{a-1} \mu_{n}(x)}{\left(\theta-\theta_{1}\right)^{a}}+\mu(x, \theta),
$$

where $\mu_{1}, \mu_{2}, \ldots$ do not involve $\theta$ and $\mu$ does not become infinite when $\theta=\theta_{1}$.

Hence

$$
\begin{aligned}
g(x) & =(1-\theta M) f(x) \\
& =\Sigma \frac{\theta^{t-1}}{\left(\theta-\theta_{1}\right)^{4}}(1-\theta M) \mu_{q}(x)+(1-\theta M) \mu(x, \theta) \\
& =\Sigma\left[\frac{\theta^{4}}{\left(\theta-\theta_{1}\right)^{\prime \prime}}\left(\frac{1}{\theta_{1}}-M\right) \mu_{l}(x)-\frac{\theta^{\prime-1}}{\left(\theta-\theta_{1}\right)^{q-1}} \frac{1}{\theta_{1}} \mu_{l l}(x)\right]+(1-\theta M) \mu(x, \theta) .
\end{aligned}
$$

Since the residues here must vanish, we have

$$
\begin{aligned}
& \left(1-\theta_{1} M\right) \mu_{i}(x)=0, \\
& \left(1-\theta_{1} M\right) \mu_{q}(x)=\mu_{q+1}(x) \quad(q=1,2, \ldots, a-1) . \\
& \quad \mu_{1}(x)=\left(1-\theta_{1} M\right)^{q-1} \mu_{1}(x),
\end{aligned}
$$

Thus

and $\mu_{1}(x)$ is destroyed by the operator $\left(1-\theta_{1} M\right)^{a}$; it is therefore a linear combination of the functions $h, h^{\prime}, h^{\prime \prime}, \ldots$ of $\S 8 a$ and since

$$
\left(1-\theta_{1} M\right)^{a-1} \mu_{1}(x)=u_{\alpha}(x)
$$

and does not vanish, $a$ cannot exceed the number of members in the longest associated sequence.*

The equation (26) now becomes

$$
g(x)+\mu_{1}(x) / \theta_{1}=(1-\theta M) \mu(x, \theta) .
$$

Since the left side does not involve $\theta$,

$$
(1-\theta M) \mu(x, \theta)=\left(1-\theta_{1} M\right) \mu\left(x, \theta_{1}\right),
$$

* That is, a sequence of functions $h, h^{\prime}, h^{\prime \prime}, \ldots$ associated with $\theta_{1}$, such that and so on as in 8 .

$$
\left(1-\theta_{1} M\right) h=0, \quad\left(1-\theta_{1} M\right) h^{\prime}=h,
$$


whence it follows that $\mu(x, \theta)=\left(1-\theta_{1} M\right) \mu^{\prime}(x, \theta)$,

where

$$
\mu^{\prime}(x, \theta)=\left\{\theta_{1} \mu\left(x, \theta_{1}\right)-\theta \mu(x, \theta)\right\} /\left(\theta_{1}-\theta\right),
$$

and does not become infinite when $\theta=\theta_{1}$.

Hence

$$
g(x)+\mu_{1}(x) / \theta_{1}=(1-\theta M)\left(1-\theta_{1} M\right) \mu^{\prime}(x, \theta)=\left(1-\theta_{1} M\right)^{2} \mu^{\prime}\left(x, \theta_{1}\right)
$$

similarly, and this again may be written

where

$$
\begin{gathered}
(1-\theta M)\left(1-\theta_{1} M\right)^{2} \mu^{\prime \prime}(x, \theta), \\
\mu^{\prime \prime}(x, \theta)=\left\{\theta_{1} \mu^{\prime}\left(x, \theta_{1}\right)-\theta_{\mu^{\prime}}(x, \theta)\right\} /\left(\theta_{1}-\theta\right) .
\end{gathered}
$$

This process can be carried on indefinitely, so that the equation

$$
\left(1-\theta_{1} M\right)^{p} f(x)=g(x)+\mu_{1}(x) / \theta_{1}
$$

is soluble for all positive integral values of $p$. The conditions for this were given in $\S 8 a$, and enable us to find $\mu_{1}(x)$ : then $\mu_{q}(x)$ is given by (29) and the residues in $f(x)$ are completely determined.

In particular, if $g$ has the value $h^{(a)}$, belonging to a sequence $h, h^{\prime}, h^{\prime \prime}, \ldots$, we have

$$
\mu_{1}=-\theta_{1} h^{(q)}, \quad \mu_{2}=-\theta_{1} h^{(q-1)}, \quad \ldots, \quad \mu_{l+1}=-\theta_{1} h:
$$

thus $a$ is at least $q+1$, that is, the longest sequence cannot have more than $\alpha$ members : it has been proved already that it cannot have fewer, and hence $a$ is the number of members in the longest sequence. Since $\alpha$ must be finite, so must all the sequences be.

If the sequences are in canonical form and that to which $h^{(2)}$ belongs contains $\beta$ members, $\mu_{1}(x)$ must in general contain the term

$$
-\theta_{1} h^{(q)}(x) \int_{0}^{1} g(x) k^{(\beta-q-1)}(x) d x / \int_{0}^{1} h^{(q)}(x) k^{(\beta-1-1)}(x) d x .
$$

The part of $f(x)$ that becomes infinite when $\theta=\theta_{1}$ is then the aggregate of such expressions as

$$
\begin{aligned}
& -\frac{\int_{0}^{1} g(x) k^{(\beta-1-1)}(x) d x}{\int_{0}^{1} h^{(q)}(x) k^{(\beta-q-1)}(x) d x} \\
& \times\left[\frac{\theta_{1} h^{(q)}(x)}{\theta-\theta_{1}}+\frac{\theta \theta_{1} h^{(q-1)}(x)}{\left(\theta-\theta_{1}\right)^{2}}+\frac{\theta^{2} \theta_{1} h^{(1-2)}(x)}{\left(\theta-\theta_{1}\right)^{3}}+\ldots+\frac{\theta^{\prime} \theta_{1} h(x)}{\left(\theta-\theta_{1}\right)^{\prime+1}}\right],
\end{aligned}
$$

there being one such for every member of every sequence.*

* This form was substantiaily given by me (Camb. Phil. Trans., Vol. xix., Part 2, p. 203). Compare also Plemelj (Monatshefte f. Math. u. Physik, 1904), and the papers of Mercer and Heywood. 
12. The expression in square brackets is the value of $f(x)$ that satisfies (1) when $g(x)$ has the value $-h^{(y)}(x)$, and this is true even when $\theta$ has another of the values for which $\Delta$ vanishes, say $\theta_{2}$. Thus the equation

$$
\left(1-\theta_{2} M\right) f(x)=h^{(4)}(x)
$$

is soluble for all the values of $q$, and, as its solution involves only the functions of the sequence,

$$
\left(1-\theta_{2} M\right)^{2} f(x)=h^{(\gamma)}(x)
$$

is also soluble, and in general so is

$$
\left(1-\theta_{2} M^{p} f(x)=h^{(q)}(x) .\right.
$$

Hence, if $k_{2}, k_{2}^{\prime}, \ldots$ is a sequence of functions associated with $\theta_{2}$, we have

$$
\int_{0}^{1} h^{(q)}(x) k_{2}^{(r)}(x) d x=0
$$

for all the values of $q, r$.

The determination of the zeros of $\Delta$ and the associated sequences of functions corresponds exactly to the reduction of an algebraic matrix to its canonical form, for we have

$$
\begin{array}{rlrl}
\theta_{1} M h(x) & =h(x), & & \theta_{1} \bar{M} k(x)=k(x), \\
\theta_{1} M h^{\prime}(x) & =h^{\prime}(x)-h(x), & & \theta_{1} \bar{M} k^{\prime}(x)=k^{\prime}(x)-k(x), \\
\theta_{1} M h^{\prime \prime}(x) & =h^{\prime \prime}(x)-h^{\prime}(x), & & \text { and so on. } \\
\quad \text { and so on } ; & &
\end{array}
$$

13. As functions of the complex variable $\theta$, whose singularities in the tinite part of the plane are known, $f(x)$ and the solving function ${ }^{*} \psi(x, y)$ can often be expressed in series of partial fractions, with possibly an added integral function.

When there are only poles of the first order the expansions are in the simplest case

$$
\sum a_{r} \theta_{r} h_{r}(x) /\left(\theta_{r}-\theta\right) \quad \text { and } \sum A_{r} h_{r}(x) k_{r}(y) /\left(\theta_{r}-\theta\right),
$$

where

$$
A_{r}=1 / \int_{0}^{1} h_{r}(x) k_{r}(x) d x
$$

and

$$
a_{r}=A_{r} \int_{0}^{1} g(x) k_{r}(x) d x .
$$

* $\psi$ is given by the equation $\left(1-\theta I_{x}\right) \psi(x, y)=\phi(x, y)$

and is such that

$$
f(x)=g(x)+\theta \int_{0}^{1} \psi(x, y) g(y) d y .
$$


For the particular value 0 of $\theta$ we have

$$
g(x)=\Sigma a_{r} h_{r}(x), \quad \phi(x, y)=\Sigma A_{r} h_{r}(x) k_{r}(y) / \theta_{r} .
$$

if the expansions are valid. This question is too wide* to be treated in an appendix to a discussion on integral equations, but a few remarks may be made without any claim to novelty.

If the expansion $g(x)=\Sigma \alpha_{r} h_{r}(x)$ holds, and may be integrated term by term, then, since

$$
\begin{aligned}
& \left(\theta_{r}-\theta\right) \int_{0}^{1} h_{r}(y) \psi(x, y) d y \\
& =\theta_{r} \int_{0}^{1} \psi(x, y)\left(1-\theta M_{y}\right) h_{r}(y) d y=\theta_{r} \int_{0}^{1} h_{r}(y)\left(1-\theta \bar{M}_{y}\right) \psi(x, y) d y \\
& =\theta_{r} \int_{0}^{1} h_{r}(y) \phi(x, y) d y=h_{r}(x),
\end{aligned}
$$

we have $f(x)=g(x)+\theta \Sigma \alpha_{r} \int_{0}^{1} h_{r}(y) \psi(x, y) d y$

$$
=g(x)+\theta \Sigma \alpha_{r} h_{r}(x) /\left(\theta_{r}-\theta\right)=\Sigma a_{r} \theta_{r} h_{r}(x) /\left(\theta_{r}-\theta\right) .
$$

On the other hand, if the expansion

$$
\phi(x, y)=\Sigma A_{r} h_{r}(x) k_{r}(y) / \theta_{r}
$$

holds and may be integrated term by term, we have, by the same method,

$$
\psi(x, y)=\Sigma A_{r} h_{r}(x) k_{r}(y) /\left(\theta_{r}-\theta\right)
$$

and

$$
\begin{aligned}
f(x) & =g(x)+\theta \Sigma A_{r} h_{r}(x) \int_{0}^{1} g(y) k_{r}(y) d y /\left(\theta_{r}-\theta\right) \\
& =g(x)+\theta \Sigma \alpha_{r} h_{r}(x) /\left(\theta_{r}-\theta\right) .
\end{aligned}
$$

Again, if the series $\Sigma a_{r} h_{r}(x)$ converges and may be integrated term by term, but its sum is not $g(x)$, the series $\Sigma a_{r} \theta_{r} h_{r}(x) /\left(\theta_{r}-\theta\right)$ will also converge, since it may be written

$$
\Sigma \alpha_{r} h_{r}(x)+\theta \int_{0}^{1}\left\{\Sigma \alpha_{r} h_{r}(y)\right\} \psi(x, y) d y .
$$

Thus

$$
f(x)-\Sigma a_{r} \theta_{r} h_{r}(x) /\left(\theta_{r}-\theta\right)
$$

is an integral function of $\theta$ and equal to a series of ascending powers of $\theta$ convergent in all the plane. The operation $1-\theta M$ upon this expression gives the result $g(x)-\Sigma \alpha_{r} h_{r}(x)$, say $g^{\prime}(x)$, and the series must be

$$
g^{\prime}(x)+\theta M g^{\prime}(x)+\theta^{2} M \Gamma^{2} g^{\prime}(x)+\ldots,
$$

so that, as $n$ increases, $M^{n} g^{\prime}(x)$ must ultimately decrease faster than the $n$-th power of any fraction whatever.

* It includes the whole theory of Fourier's series. 
14. Example I.-Let

$$
\phi(x, y)=F(x-y)-F(x+y),
$$

where $F$ is a function such that

$$
F(x)=F(-x)=F(x+2),
$$

and therefore

$$
F(1-x)=F(1+x) \text {. }
$$

Then

$$
\begin{aligned}
M \sin p \pi x & =\int_{0}^{1} \phi(x, y) \sin p y \pi d y \\
& =\int_{-x}^{1-x} F(y) \sin p(y+x) \pi d y-\int_{x}^{++x} F(y) \sin p(y-x) \pi d y,
\end{aligned}
$$

and, since $\int_{-x}^{0} F(y) \sin p(y+x) \pi d y=\int_{0}^{x} F(y) \sin p(x-y) \pi d y$

and $\quad \int_{1}^{1+x} F(y) \sin p(y-x) \pi d y=\int_{1-x}^{1} F(2-y) \sin p(2-y-x) \pi d y$,

this expression becomes

or

$$
\begin{gathered}
\int_{0}^{1} F(y)\{\sin p(y+x) \pi-\sin p(y-x) \pi\} d y \\
2 \sin p \pi x \int_{0}^{1} F(y) \cos p \pi y d y ;
\end{gathered}
$$

therefore $\sin p \pi x$ is associated with a zero of $\Delta,{ }^{*}$ when $n$ is great enough (\$2).

Thus on account of the symmetry of $\phi(x, y)$ the two series of functions $h, k$ are in this case

$$
\sin x \pi, \sin 2 x \pi, \sin 3 x \pi, \ldots
$$

By taking for $F(x)$ a continuous function whose Fourier series is not always convergent, $\dagger$ we see that even when $\phi(x, y)$ is continuous, the associated series $\Sigma A_{r} h_{r}(x) k_{r}(y) / \theta_{r}$ is not necessarily always convergent.

If

$$
\int_{0}^{1} F(y) \cos p \pi y d y=0
$$

$\sin p \pi x$ will be wanting in the series $\Sigma u_{r} h_{r}(x)$, which will therefore not be able to represent any function $g(x)$ unless

$$
\int_{0}^{1} g(x) \sin p \pi x d x=0 .
$$

* The method of $\$ 2$, followed out in this case, shews that these are the only zeros of $\Delta$.

+ Hobson, Functions of a Real Variable, p. 705. 
Any number of terms may be wanting in this way, even an infinity, as would happen were $F(x)=-F(1+x)$ when only sines of odd multiples could occur.

$$
\begin{aligned}
& \text { Example II.-Take } \phi(x, y)=1 \text {, when } y<x \text {, } \\
& =0 \text {, when } y>x \text {. }
\end{aligned}
$$

Thus

$$
\begin{aligned}
& \int_{0}^{1} \phi(x, y) f(y) d y=\int_{0}^{x} f(y) d y \text {, } \\
& M_{x} \phi(x, y)=\int_{0}^{1} \phi(x, t) \phi(t, y) d t=\int_{y}^{x} d t=x-y . \quad(x>y) \\
& =0 \quad(x<y) .
\end{aligned}
$$

Similarly

$$
M_{x}^{2} \phi(x, y)=\frac{1}{2}(x-y)^{2} \quad(x>y),
$$

and so on. The solving function

$$
\begin{aligned}
\psi(x, y) & =e^{\theta(x-y)} & & (x>y) \\
& =0 & & (x<y) .
\end{aligned}
$$

The solution of (1) is $f(x)=g(x)+\theta \int_{0}^{x} e^{\theta(x-y)} g(y) d y$.

In this example there are no special functions such as $h, k$, and, since

$$
M^{n} g(x) \leqslant \frac{x^{n}}{n !} \times \text { the upper limit of } g(x),
$$

it follows that, when $g(x)$ is any limited function, $M^{n} g(x)$ diminishes, as $n$ increases, ultimately faster than the $n$-th power of any fraction, in accordance with the last result of $\S 13$.

[For a proof of the theorem of de la Vallée-Poussin and Lebesgue used on p. 319 , see a paper by the writer that is to appear shortly in the Proceedings of the Cambridge Philosophical Society.] 